\title{
Novel Methods for Hard X-ray Holographic Lensless Imaging
}

\author{
M. Saliba ${ }^{1.2}$, J. Bosgra ${ }^{3}$, A. D. Parsons ${ }^{2}$, U. H. Wagner ${ }^{2}$, C. Rau ${ }^{2}$, and P. Thibault ${ }^{2,4}$ \\ 1. University of Zürich, Department of Physics, Zürich, Switzerland. \\ 2. Diamond Light Source Ltd, I13 Beamline, Oxfordshire, United Kingdom. \\ 3. Paul Scherrer Institute, Laboratory of Micro- and Nanotechnology, Villigen, Switzerland. \\ 4. University of Southhampton, Department of Physics \& Astronomy, Southhampton, United Kingdom.
}

Holography is a coherent imaging method that provides complex-valued reconstructions of the wave field at the exit of a sample by virtue of the interference between the scattered sample wave and a reference wave [1]. Fourier transform holography (FTH) is a robust and reliable method where reconstructions are obtained by a single Fourier transform, owing to a nearby point-scatterer whose transmission function is approximated to a delta function [2]. Hence, the total autocorrelation function includes the cross-correlation between the sample function and the delta-like reference function, leading to two separated twin images of the sample. Holographic techniques emerged using different types of reference structures such as extended references [3] (slits, corner apertures, etc.), uniformly redundant arrays, and customised references [4]. Such methods have been successfully implemented using soft Xrays $[2,3,4,5]$. However, for hard $\mathrm{X}$-rays, the fabrication of high-resolution fully absorbing reference masks is almost impossible. Therefore, high-resolution holographic diffraction imaging with hard Xrays requires a solution to the fabrication limitations. Furthermore, for an absorbing mask, most methods have also employed a "monolithic" frame [5] where the reference and sample are bound on the same substrate. This limits the flexibility of the setup and inhibits independent translation and rotation.

The development of novel e-beam lithography techniques, crystal etching, and atomic layer deposition, applied for high-resolution fabrication of X-ray optics such as Fresnel zone plates (FZP), provides new horizons for creating specially tailored reference structures for hard X-ray holographic imaging. To that end, we developed new designs for a variety of references such as micro-FZPs and 3D extended references fabricated by e-beam lithography on a transmissive substrate and implemented in a holographic setup that decouples the reference from the sample. The reference design bypasses the constraints of ionbeam milling and the limitations associated with absorbing masks. One example of such references is a $10 \mu \mathrm{m}$ diameter gold FZP with a $70 \mathrm{~nm}$ outer-zone width (Figure 1.d), which converges incident light with high efficiency into the first-order focus. In a holographic setup, the sharp and intense focal spot serves as the point-source reference to provide direct reconstructions by a single Fourier transform.

The experiments are performed the I13-1 Beamline at Diamond Light Source using a flat monochromatic beam of $9.1 \mathrm{keV}$ X-rays. The reference mask constitutes of four closely arranged $10 \mu \mathrm{m}$ FZPs to increase the reference flux and provide a total of eight cross-correlation reconstructions at the front- and back-focal plane. A test-sample of $340 \mathrm{~nm}$ gold thickness is placed in the vicinity of FZP first order focal plane. A photon-counting detector is placed $14.7 \mathrm{~m}$ from the sample where the far-field holographic diffraction pattern is recorded as a result of the interference between the four divergent reference waves and the wave scattered by the sample, as shown in Figure 1a. By computing the inverse Fourier transform and applying near-field propagation, four reconstructions of the sample are brought into focus at the lateral positions corresponding to the four FZPs. Figure $1 \mathrm{~b}$ and $1 \mathrm{c}$ show the reconstructed field of view in amplitude and phase respectively, where we find four images of the cartoon gold fish and a central region of high intensity due to the intense diverging reference beams of 
the FZPs. The $150 \mathrm{~nm}$ outline of the sample and $400 \mathrm{~nm}$ gold dot (eye of the fish) are resolved, and the sub-100 nm details in the tail of the fish are distinguishable. With the fabrication of FZPs of outer zone width up to $10 \mathrm{~nm}$, the image resolution can be further enhanced. Employing an order-sorting aperture and a central stop filters out the intense background signal due to the interference of the FZP waves.

In addition, using similar fabrication techniques, three-dimensional extended references are fabricated and successfully implemented with hard X-ray holography to reconstruct weakly and strongly scattering samples with high resolution and contrast as well as low noise sensitivity by the autocorrelation differential operator. Such methods provide complex-valued reconstructions without the need for scanning the sample or iterative phase retrieval. The decoupled sample arrangement relaxes the technical constraints enabling tomographic imaging. Furthermore, the method is applicable to single-shot femtosecond imaging [6] with X-ray free-electron lasers applied to biological and dynamic specimens.

[1] D Gabor, Nature 161 (1948) p. 777-778.

[2] S. Eisebitt et al, Nature 432, (2004) p. 885-888.

[3] M. Guizar-Sicairos and J. R. Fienup, Opt. Express 15, (2007) p. 17592-17612.

[4] A. V. Martin et al, Nat. Commun. 5:4661, (2014).

[5] J. Geilhufe et al, Nat. Commun. 5:3008, (2014).

[6] C. M. Gunther et al, Nat. Photonics 5, (2014) p. 99-102.

[7] We acknowledge funding from the University of Zürich and Diamond Light Source Ltd. We especially thank Prof. Juerg Osterwalder for his consultation and supervision. We thank Dr. Christian David at the Paul Scherrer Institute for providing the fabrication methods.

a
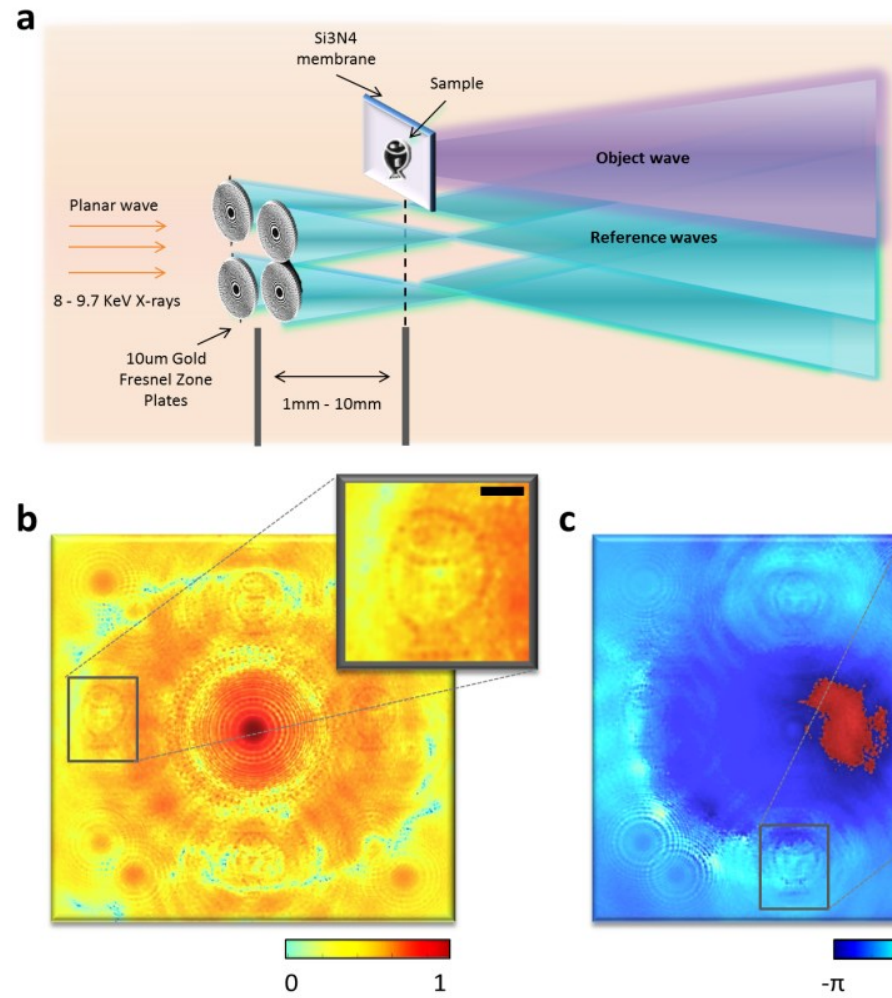
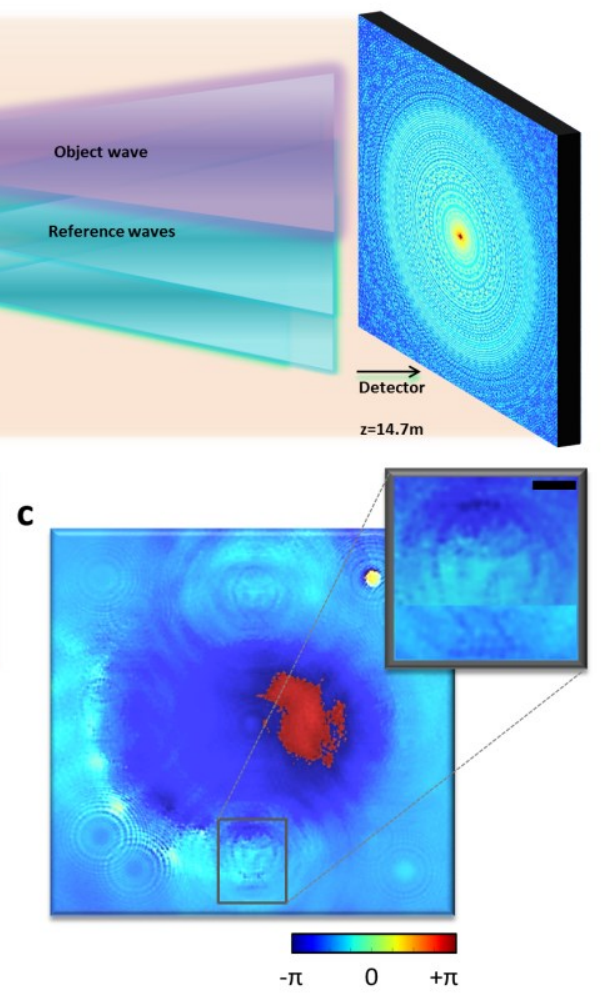
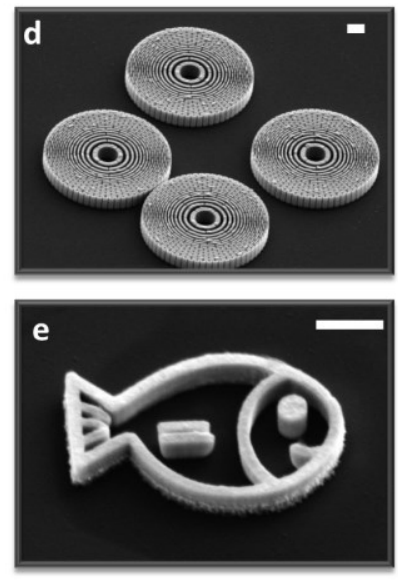

Figure 1. a. Schematic of holographic diffraction measurement. b. Amplitude reconstruction. c. Phase reconstruction. d. SEM image of reference FZP structure. e. SEM image of test sample. Scale bar $1 \mu \mathrm{m}$. 